\title{
Fatty acids and beta-cell function
}

\author{
A . Björklund' ${ }^{1}$, G. Yaney ${ }^{2}$, J.D. M cG arry 3 , G. Weir ${ }^{4}$ \\ ${ }^{1}$ Department of Molecular Medicine, Karolinska Institute, Stockholm, Sweden \\ ${ }^{2}$ Evans Department of Medicine, Boston Medical Center, Boston, Massachusetts, USA \\ ${ }^{3}$ Department of Internal Medicine, Southwestern Medical Center at Dallas, Dallas, Texas, USA \\ ${ }^{4}$ Joslin Diabetes Center, Boston, Massachusetts, USA
}

In opening remarks, J. Denis McGarry pointed out that at the Third JDF World Conference on Diabetes Research held in Monaco in 1991 there was little, if any, mention of the role of fatty acids in pancreatic betacell function. However, this situation has now changed dramatically on the heels of two recent and simultaneous developments. The first has to do with the notion that there is at work within the beta cell, just as in other tissues, an important element of glucose-fatty acid "cross-talk" which appears to be essential for the normal regulation of insulin secretion. The second revelation is that chronic exposure of the beta cell to high levels of circulating fatty acids can actually be detrimental to stimulus-secretion coupling, and might be a factor in the aetiology of non-insulin-dependent, and possibly insulin-dependent, diabetes. Thus, the emerging picture is that from the standpoint of beta-cell function fatty acids can play "a good, a bad and an ugly" role. These characteristics form the subject of the current session.

\section{Barbara Corkey: Long chain acyl-CoAs and signal} transduction in the betacell

Fuels in the beta-cell generate intracellular signals depending upon whether there is glucose or fat,

Participants: B.E. Corkey, Diabetes and Metabolism Unit, Boston University, Medical Center, Boston, Massachusetts, USA

M. Prentki, Department of Nutrition, University of Montreal, Montreal, Quebec, Canada

R.H. Unger, Department of Internal Medicine, University of Texas, Southwestern Medical Center at Dallas, Texas, USA

V. Grill, Department of Medicine, University Hospital of Trondheim, Trondheim, Norway

Corresponding author: J.D. McGarry, Ph.D., University of Texas, Southwestern Medical Center at Dallas, Internal Medicine Department, 5323 Harry Hines Boulevard, Dallas, Texas 75235-9135, USA glucose and fat or an excess of each present. Several beta cell parameters were monitored simultaneously after glucose stimulation to help establish cause and effect relationships. All of the metabolic parameters measured reached their half-maximal values before the initial change in intracellular free calcium was detected, thus providing direct evidence that metabolic changes preceded changes in calcium in beta-cell signalling [1]. These assumptions provide the underpinning for a model of stimulus-secretion coupling [2] in which phosphorylation of transported glucose causes an initial drop in the ATP/ADP ratio which results in the stimulation of mitochondrial respiration and the activation of phosphofructokinase (PFK)-1, ultimately bringing about an increase in the ATP/ADP ratio. This increase would reduce the activity of the $\mathrm{K}^{+}$ATP channels, depolarize the cell, and open voltage-dependent calcium channels, resulting in a rise in intracellular free calcium and stimulation of insulin secretion.

However, recent work by Henquin and co-workers has provided evidence that glucose can generate signals without changes in electrical activity or calcium movement. Based on the work of several groups, including this one [3], normal secretion from the beta cell is known to be oscillatory in nature. Corkey and co-workers have shown that even under "clamped" conditions these oscillations still persist with the same amplitude and period. What this suggests is that glucose metabolism generates signals that are driving oscillations in calcium and that these changes in intracellular calcium are not the only trigger for stimulated secretion, but may be permissive for the action of other signals generated.

The long-chain fatty acyl-CoA esters (LC-CoA) are hypothesized to be one of these other signals. It is believed that these rise in the cytosol through the action of acetyl-CoA carboxylase (ACC) to produce malonyl-CoA which is an important "switch" 
compound, inhibiting the LC-CoA transporter, carnitine palmitoyltransferase I (CPT-I), and preventing fatty acid oxidation. $\mathrm{LC}-\mathrm{CoA}$ is proposed to interact with effector proteins to initiate stimulated secretion. Addition of exogenous non-esterified fatty acids (NEFA), which are activated in the cell to LC-CoA, stimulates secretion. The potential of LC-CoA as an effector molecule is supported by its multiple cellular sites of action, including: glucokinase and glucose 6phosphatase, certain steps in exocytosis, fusion events at the Golgi, calcium handling at the endoplasmic reticulum, various isoforms of protein kinase $\mathrm{C}$, the enzymes CPT-I and ACC, and various effects at the level of gene expression.

The actions of LC-CoA on PKC isoforms, exocytosis, and the ATP-sensitive $\mathrm{K}^{+}$-channel were highlighted by work from Corkey and collaborators. The isoforms of PKC are divided into three classes depending on the lipids needed for activation. Based on activities in whole homogenates of HIT cells, oleyl-CoA can activate the atypical and classical isoforms, while inhibiting the calcium-independent novel isoforms. Using permeabilized HIT cells as a model, elevating the ambient calcium concentration was shown to increase insulin secretion, and the addition of palmitoyl-CoA enhanced this secretion at all levels of calcium. Interestingly, this stimulation by LC-CoA was greater at the higher calcium levels.

Recent work in isolated inside-out patches of ob/ob islet cells (95\% beta cells) has shown that LC-CoA also affects $\mathrm{K}^{+}{ }_{\text {ATP }}$ channel activity [4]. As expected, ATP inhibited the channel activity, while oleic acid or free CoA, as well as short chain acyl-CoAs, such as malonyl-CoA, were without effect. Unexpectedly, the addition of oleyl-CoA opened the channel in a concentration dependent fashion, peaking at $1 \mu \mathrm{mol} / \mathrm{l}$. The ability of oleoyl-CoA to open the channel was seen in the presence of ATP and ADP. Indeed, simultaneous addition of $\mathrm{Mg}^{2+}$-ADP and LC-CoA synergized to dramatically increase mean open time of this channel. This has led to the proposal of a model in which $\mathrm{Mg}^{2+}$-ADP and LC-CoA bind allosterically to the regulatory component of this channel, with the long chain acyl group perhaps serving to anchor the regulatory subunit at the plasma membrane. Although opening of the channel by oleyl-CoA appears counterintuitive, it must be remembered that glucose metabolism oscillates. It is proposed that oscillatory increases in LC-CoA are responsible for the observed oscillatory opening of the $\mathrm{K}^{+}{ }_{\text {ATP }}$ channel, while oscillatory decreases in $\mathrm{Mg}^{2+}$-ATP cause closure.

The addition of either the adenyl cyclase activator, forskolin, or the intestinally secreted incretin, glucagon-like peptide (GLP)-1, raises intracellular cAMP and augments stimulated insulin secretion. How does this come about? It is suggested here that the mechanism could involve the release of intracellular NEFA by lipolysis. In clonal beta cells, as expected, glucose inhibited NEFA conversion to $\mathrm{CO}_{2}$. In contrast, addition of GLP-1 caused a twofold increase in NEFA oxidation which could still be inhibited by high glucose. Since NEFA caused acidification, as measured by an intracellular probe, BCECF, a drop in $\mathrm{pH}$ occurred with release of internal NEFA or the addition of exogenous NEFA. Changes in NEFA in the medium can be measured by the extracellular probe, ADAFAB. GLP-1 was shown to decrease the $\mathrm{pH}$ while increasing extracellular NEFA. These effects were amplified by addition of Triacsin-C, which prevents activation of NEFA to their CoA esters. Triacsin- $\mathrm{C}$ did not block the glucose-stimulated rise in $\mathrm{pH}$ or insulin secretion, but only the incremental potentiation provided by GLP-1. Consistent with the proposed stimulation of lipolysis in the beta cell, the presence of hormone-sensitive lipase was documented using the polymerase chain reaction (RT-PCR).

Finally, recent work in Dr. Corkey's laboratory suggests that the mitochondrial uncoupling protein, UCP-2, is also present in clonal beta cells and may play a role in modulating respiration. Data indicated that glucose doubled $\mathrm{O}_{2}$ consumption, while forskolin gave a rise of approximately 2.5-fold. Measurement of pyridine nucleotide fluorescence showed that whereas glucose produced a sharp increase in redox state, forskolin caused an oxidation. It is hypothesized that NEFA mobilized by the rise in cAMP activate UCP-2, thus stimulating respiration and increasing the amplitude of oscillations observed in insulin secretion. The presence of UCP-2 transcript in these cells was confirmed by RT-PCR.

In conclusion, it is proposed that fatty acids play an important role in signal transduction either directly or by conversion to their CoA esters. Levels of these important molecules change in response to glucose, exogenous or endogenous NEFA, as well as agents that elevate intracellular cAMP.

\section{Marc Prentki: Fatty acid regulation of metabolic enzyme gene expression in the beta cell}

While the previous speaker addressed the acute role of LC-CoA in beta-cell signal transduction, this talk illustrated some of the long term effects of fatty acids on beta-cell function. The basic glucose signal transduction outlined was much the same as that presented by Dr. Corkey. Glucose is metabolized to pyruvate, but from pyruvate the pathway splits into two arms; one may be called the acetyl-CoA $/ \mathrm{K}^{+}$ATP pathway and the other the anaplerosis/malonyl-CoA pathway. Activation of the former leads to a rise in the ATP/ADP ratio, cell depolarization and an increase in intracellular free calcium. Increased flux through the second arm leads to elevation of the cytosolic citrate concentration and, through the action of acetyl-CoA carboxylase (ACC), to increased levels 
of malonyl-CoA, inhibition of carnitine palmitoyltransferase I (CPT-I) and an increase in cytosolic LC-CoA species. Input from both arms is required for sustained secretion by nutrient secretagogues. In addition, because these pathways respond to various fuels to generate "signals of plenty", such as malonyl-CoA and an increased ATP/ADP ratio, they are more than glucose-sensing, but help to integrate various inputs. In this way, the mode of action of all calorigenic nutrient stimuli, such as carbohydrates, amino acids, and fatty acids, to increase insulin secretion can be explained [5].

This model provides the framework for what may partially explain beta-cell insensitivity to glucose after exposure to increased levels of fatty acids, as might occur with obesity. Under these conditions, there appears to be disruption of normal fuel partitioning within the cell by alteration in the levels of two key enzymes: ACC and CPT-I. This is critical in the beta cell, for these enzymes are not normally used to store triglycerides, but to switch the cell from the oxidation of lipids to their esterification as part of glucose signal transduction. Consistent with this idea is the fact that fatty acid synthase (FAS) activity is very low in this cell type. The model cell line used here, INS-1, is glucose responsive and has been proven to mimic the altered response of islets after prolonged exposure to elevated lipids, resulting in an increased basal insulin secretion and a blunted response to stimulation by glucose.

A CC gene regulation: Stimulatory levels of glucose increase the mRNA transcript for this enzyme. Exposure to linoleate reduced the transcript level within $3 \mathrm{~h}$ and the effect was maximal at $12 \mathrm{~h}$ under both high and low glucose conditions [6]. This effect requires the oxidation of the fatty acid, because bromopalmitate, an unmetabolizable fatty acid analogue, is not effective. Both saturated and unsaturated fatty acids reduced the ACCmRNA level, as did the short chain species, butyrate and caproate. However, this was not true of the omega-3 fatty acids. It is important to note that the effective fatty acids described here are the most common naturally occurring species and have been used in these experiments at physiologically relevant concentrations. In addition, it was shown that exposure to oleate caused a proportional reduction in both ACC mRNA and malonyl-CoA levels. The mechanism involved in downregulating this mRNA is not known, but it may be secondary to the metabolism of fatty acid or the concomitant reduction in glucose oxidation.

CPT-I gene regulation: There is a rapid and substantial induction of the CPT-I transcript by NEFA within $1 \mathrm{~h}$ of exposure to palmitate as well as bromopalmitate, both responses peaking at $6 \mathrm{~h}$ [7]. Interestingly, in the case of bromopalmitate the level of induction appears to be stable up to $24 \mathrm{~h}$ while the response to palmitate falls off after $6 \mathrm{~h}$, perhaps due to metabolism of the natural fatty acid. The effectiveness of bromopalmitate indicates that, unlike the modulation of ACC mRNA, for the induction of CPT-I the fatty acids do not have to be metabolized. Therefore, there appear to be at least two mechanisms involved in modulating gene transcription. This is corroborated by nuclear run-on studies which indicated that induction is at the level of transcription and that mRNA stability is not affected. In addition, induction is seen in the presence of cycloheximide, indicating activation by pre-existing transcriptional factors. As is seen with many early-response genes, the level of induction is actually exaggerated in the presence of this inhibitor.

As a functional confirmation of CPT-I gene induction, there was a time-dependent increase in its activity in INS- 1 cells after the addition of oleate. The oxidation of fatty acids was also enhanced. It is suggested that because of these changes there was now a "Randle cycle" established in the altered cells where one had not existed before, and that this change would explain the blunted response of insulin secretion to glucose stimulation. Since CPT-I serves as a master gene controlling fuel partitioning in cells, it would be a logical candidate gene for involvement in the induction of NIDDM.

\section{J. Denis McGarry: Influence of structure on the insulinotropic potency of fatty acids}

At this point in the proceedings Dr. McGarry made a cameo presentation on some recent work his group had done regarding the insulinotropic effect of NEFA in the rat. Three key points emerged. First, when the elevated level of plasma NEFA in the intact fasted rat is suppressed by infusion of nicotinic acid (NA), insulin secretion in response to an intravenous glucose challenge is totally ablated. Second, when the NEFA level is maintained high in NA-treated animals by coinfusion of Intralipid plus heparin glucosestimulated insulin secretion (GSIS) becomes supranormal [8]. Third, not all fatty acids are "made equal" in terms of their ability to enhance GSIS, this property being profoundly influenced by their chain length (positively) and degree of unsaturation (negatively).

Although it is not yet clear how fatty acids exert such dramatic, physiological effects on GSIS, these findings bring to light a hitherto unrecognized principle of pancreatic beta-cell function and fuel homeostasis, namely, that in the fasted rat, were it not for the elevated plasma NEFA concentration the beta cell would be blind to glucose when the fast is terminated. They also indicate that at any given time the beta-cell's response to glucose in terms of insulin secretion will be strongly influenced not only by the 
total concentration of NEFA in plasma, but also by the nature of the NEFA mix [9]. This could have important implications for understanding the suspected linkage between dietary saturated fat and the genesis of hyperinsulinaemia, dyslipidaemia and insulin resistance.

Roger Unger*: Mechanisms of beta-cell dysfunction: the role of intracellular triglyceride stores

In human obesity increases in insulin resistance are at first unaccompanied by hyperglycaemia because of a precise compensatory increase in insulin secretion by beta cells of the islets of Langerhans. However, late in the course of obesity compensatory hyperinsulinaemia may wane and hyperglycaemia may appear, and adipogenic NIDDM is diagnosed. The mechanisms involved in the decompensation of the beta cell are poorly understood, but one potential mechanism involves elevated intracellular triglyceride (TG) stores, since these are increased in plasma and tissues of obese subjects.

The Zucker diabetic fatty (ZDF) rat is a good model of human obesity. As in humans, obesity begins early in life, and is associated with insulin resistance that is precisely compensated for by increased insulin secretion. At about 9 weeks of age, however, compensation begins to decrease and hyperglycaemia results. In the compensated pre-diabetic ZDF beta cell TG stores are increased threefold compared to lean ZDF littermates. This difference increases to 10 -fold in the decompensated beta cell. Longitudinal studies in pre-diabetic ZDF rats revealed that the latter elevation in islet fat content coincided precisely with the onset of hyperglycaemia. That this increase was mechanistically relevant was suggested by in vitro studies that demonstrated that normal or compensated islets chronically exposed to NEFA developed compensated and decompensated beta-cell phenotypes, respectively. One mechanism of increased intracellular TG stores appears to be an increase in the esterification capacity of the beta cell, as demonstrated by an increase in the mRNA and activity of glycerol phosphate acyltransferase, the first enzyme in the esterification pathway.

The increase in esterification capacity and triglyceride stores may be due to the genetic defect underlying the diabetic phenotype of the ZDF rat. This mutation has recently been identified as a defect in the leptin receptor $(\mathrm{Ob}-\mathrm{R})$ that is predominantly expressed in the hypothalamus, but is also present in islets and beta-cell lines and many other tissues. In vitro studies in normal islets demonstrated that leptin could decrease intracellular TG stores, increase NEFA oxidation and block the increase in NEFA esterification and TG accumulation associated with chronic incubation with NEFA. As expected, leptin was unable to decrease TG content or esterification capacity of islets isolated from homozygote (diabetic) ZDF animals but was able to reduce TG levels in heterozygote and wild-type (lean) ZDFs (neither of which develops diabetes).

The results of the above studies suggest that leptin regulates intracellular fat metabolism in non-adipocytes, while in leptin resistant diabetic ZDF animals this control is lost, leading to increases in intracellular TG stores, and possibly to lipotoxicity. To further address this possibility normal Wistar rats were made chronically hyperleptinaemic by infusion of an adenovirus containing the leptin cDNA. After 7 days of hyperleptinaemia all visible body fat had disappeared and the triglyceride content of islets, liver, and skeletal muscle was virtually unmeasurable. Homozygote ZDF animals were unaffected by hyperleptinaemia, as evidenced by maintenance of adipose mass and intracellular TG levels. These data support the hypothesis that the leptin receptor defect in the diabetic ZDF rat leads to an excessive accumulation of intracellular TGs. This in turn may lead to the disablement and subtotal destruction of beta cells.

One potential mechanism for the partial destruction of the beta cell in the ZDF rat is an increase in the expression of the inducible isoform of nitric oxide synthase (iNOS), leading to elevated levels of nitric oxide (NO) derivatives that ultimately result in cell death. iNOS is increased in the decompensated beta cell of the ZDF rat. Additionally, NEFA induce the expression of iNOS and NO accumulation in islets from normal Wistar rats, and islets from heterozygote and homozygote ZDFs. The NEFA induced increase is most pronounced in the diabetic homozygote. Importantly, the inhibitors of iNOS expression, nicotinamide and aminoguanidine, inhibit the FFA-induced increase in $\mathrm{NO}$ in vitro, while daily injections of these compounds prevent the diabetes of the ZDF rat in vivo. In these animals all components of the diabetic beta-cell phenotype were prevented, and the islets were large, free of fibrosis and morphologically healthy.

Since a prevailing hypothesis for the pathogenesis of autoimmune (type 1) diabetes is that cytokines induce iNOS expression, NO production, and beta-cell death, an important conclusion from these studies is that despite striking differences between immunogenic and adipogenic diabetes, the two conditions may share an overlapping mechanism for disabling and destruction of the beta cell. In islets from wildtype and homozygous ZDFs, NEFA were able to substantially increase the ability of interleukin (IL)- $1 \beta$ to induce NO production, with the greater effect occurring in homozygote islets, possibly due to their greater TG load. Additionally, the cytotoxic effect of IL- $1 \beta$ was ablated in islets isolated from hyperleptinaemic Wistar rats that were lacking measurable levels of intracellular TGs. Finally, there is a 
strong correlation between islet TG content and NO production in wild-type, heterozygous, homozygous, and hyperleptinaemic normal Wistar rats. These data strongly suggest that intracellular TGs may play a role in the disablement and destruction of the beta cell in both adipogenic and immunogenic diabetes. In adipogenic diabetes increased intracellular TG increases NO production while in immunogenic diabetes elevated intracellular TG may increase the intensity of NO-mediated autoimmune beta-cell destruction. Interventions that reduce intracellular TG (hyperleptinaemia) ablate the ability of IL- $1 \beta$ to induce NO production, suggesting that it may be possible to postpone or prevent autoimmune destruction of the beta cell by lowering the intracellular content of fat.

* This talk was given by Dr. Robert O'Doherty on behalf of Dr. Unger.

\section{Valdemar Grill: Lipotoxicity in vitro}

It was documented many years ago that NEFA are an important fuel for beta cells and that acute exposure of pancreatic islets to NEFA stimulates insulin secretion. However, the effects of long term exposure to NEFA have been much less studied, despite the fact that these effects are very relevant to conditions with elevated NEFA, such as fasting and diabetes.

Sako and Grill initially showed that infusion of lipids for $48 \mathrm{~h}$ into normal rats time-dependently inhibited glucose-induced insulin secretion after 6-24 h of NEFA exposure [10]. Similar results were later obtained in vitro by culturing normal rat islets for $48 \mathrm{~h}$ in palmitate, oleate or octanoate [11].

The role of fatty acid oxidation behind inhibition of glucose-induced insulin secretion by NEFA was studied in experiments with etomoxir. This drug inhibits fatty acid oxidation by inhibiting the carnitine palmitoyltransferase I (CPT-I) enzyme. It was found that etomoxir significantly reversed the negative influence of a $48 \mathrm{~h}$ exposure of normal rat islets to palmitate or oleate. Etomoxir did not affect the decrease in insulin release induced by the medium-chain NEFA, octanoate. The latter results were predictable, since octanoate enters mitochondrial metabolism without involvement of CPT-I. The results with etomoxir indicate linkage of the negative effects of NEFA to their metabolism. It was further shown that exposure to palmitate or oleate inhibited glucose oxidation and protein biosynthesis in pancreatic islets and that these effects were also reversed by etomoxir. The inhibition of islet glucose oxidation after long term exposure to NEFA is consistent with the operation of a glucose-fatty acid cycle as originally proposed for other tissues by Randle and co-workers.

The hypothesis that a time-dependent suppression of the pyruvate dehydrogenase (PDH) complex is
Table 1. Stimulation by fatty acids of PDH-kinase activity: short and longer term effects

\begin{tabular}{ll}
\hline Short term & Longer term \\
\hline Increased ratios of mitochon- & Increase in PDH-kinase \\
drial acetyl- $\mathrm{Co}_{\mathrm{a}} / \mathrm{CoA}$, & specific activity \\
$\mathrm{NADH}_{2} / \mathrm{NAD}$ and ATP/ADP & \\
\hline
\end{tabular}

involved in the NEFA effect was tested by exposing rat islets to palmitate for $48 \mathrm{~h}$. This resulted in a decrease in active PDH (unphosphorylated) as well as PDH total activity (tested after phosphatase treatment). The PDH kinase activity (which inactivates PDH) was simultaneously elevated, the increased activity being mainly in the free as opposed to the PDH-bound fraction of the enzyme. The importance of altered PDH kinase activities could theoretically be tested with an inhibitor of this enzyme, dichloroacetate. However, during 48 h of culture dichloroacetate inhibited glucose-induced insulin secretion and glucose oxidation in control islets, while not affecting secretion or oxidation when islets were co-cultured with palmitate. These equivocal findings invalidate the use of dichloroacetate for testing the importance of PDH kinase for islet glucose oxidation.

Long term exposure to elevated NEFA leads to a build-up of islet triglyceride stores. The longer term effects of fatty acids, as tested in vitro, could be due to massive amounts of these substrates being formed rapidly by lipolysis of islet triglycerides and then being oxidized. Possibly, a high rate of $\beta$-oxidation would depress glucose oxidation whereas a low rate would not. Alternatively, the effect of a given amount of fatty acid being oxidized could have changed due to the previous fatty acid exposure. The second possibility was tested by first pre-exposing islets to palmitate, then testing the effects of $5 \mathrm{mmol} / \mathrm{l}$ octanoate in the simultaneous presence of etomoxir (which was used to block the contribution of fatty acids derived from intracellular triglyceride stores). It was found that octanoate inhibited glucose oxidation, whereas no inhibition was seen under control conditions (after culture without palmitate). These results are compatible with a time-dependent change in the effect of fatty acid oxidation on PDH activity. Such an effect could be due to a time-dependent increase in PDH kinase activity which was previously shown to occur in non-islet tissue during fasting and diabetes. Dr. Grill envisaged that this longer term action of NEFA would act in concert with the short term effects exerted by products of $\beta$-oxidation which have been documented in other tissues (Table 1).

The role of elevated NEFA during fasting conditions was tested in normal rats that were fasted for $48 \mathrm{~h}$. Serum NEFA levels were increased by fasting and glucose-induced insulin release from isolated islets was decreased. The attenuation in insulin release was partially reversed by etomoxir. Fasting decreased 
islet PDH activity and increased PDH kinase activity. The latter effect was not associated with any increase in alpha chain of the $\mathrm{PDH}$-kinase $45 \mathrm{kD}$ protein, indicating that it was due to an increase in specific activity of PDH kinase. Altogether, these results were taken to support a role for elevated NEFA in the impairment of insulin secretion during fasting, i.e. through their ability to suppress PDH activity.

As to an influence of elevated NEFA in diabetes, experiments using human islets showed that long term culture with NEFA exerted a negative influence on glucose-induced insulin secretion which was additive to the detrimental effects of culture with elevated glucose. The importance of NEFA in diabetes was further tested in the $\mathrm{db} / \mathrm{db}$ mouse [12] which is an animal model of NIDDM combined with obesity. In $d b / d b$ mice NEFA were significantly elevated compared to non-diabetic $\mathrm{db} /+$ mice. The severely reduced glucose-induced insulin secretion in islets from $d b / d b$ animals was partially restored by etomoxir. The drug also increased the glucose oxidation to utilization ratio as well as PDH activity in $\mathrm{db} / \mathrm{db}$ islets, whereas PDH kinase activity was decreased.

All in all, the results indicate that impaired glucose-induced insulin secretion in NIDDM can to a significant extent be due to negative effects of elevated NEFA levels on PDH activity. The effects of "lipotoxicity" are additive to those of chronic hyperglycaemia. Clinical studies in NIDDM patients are now urgently needed to delineate the importance of "glucotoxicity" compared to "lipotoxicity" on beta cell function.

Finally, Dr. Grill hypothesized that, teleologically, the inhibitory effects of NEFA on insulin secretion during fasting have evolved to protect against hypoglycaemia. When NEFA are chronically elevated, as in diabetes, the same mechanisms may operate, but in this case they will be inappropriate and may damage the beta cells.

\section{Future directions and recommendations}

Although the basic studies summarized above are important in their own right, it is the translation of bench research into clinical approaches that will directly aid those with diabetes. What has become clear from the work described here is that any application of the knowledge concerning the function of the beta cell without first considering the acute and long term effects of fatty acids will be less than optimal. In that spirit, we have listed what we consider to be promising future areas of investigation.

- Learn more about the pathophysiology of diabetes and insulin resistance.
- Learn more about the interaction between diabetes and obesity.

- Attempt to develop new and effective drugs to normalize abnormally high levels of adipose tissue lipolysis and inter-tissue fatty acid flux.

- With regard to the designing of surrogate beta cells for the treatment of IDDM, keep in mind the desirable and undesirable effects of fatty acids on their function.

- Look for gene candidates contributing to the induction of IDDM and NIDDM.

\section{References}

1. Civelek VN, Deeney JT, Kubik K, Schultz V, Tornheim K, Corkey BE (1996) Temporal sequence of metabolic and ionic events in glucose-stimulated clonal pancreatic $\beta$-cells (HIT). Biochem J 315: 1015-1019

2. Prentki M, Corkey BE (1996) Are the $\beta$-cell signaling molecules malonyl-CoA and cytosolic long-chain acyl-CoA implicated in multiple tissue defects of obesity and NIDDM? Diabetes 45: 273-283

3. Cunningham BA, Deeney JT, Bliss CR, Corkey BE, Tornheim K (1996) Glucose-induced oscillatory insulin secretion in perfused rat pancreatic islets and clonal $\beta$-cells (HIT). Am J Physiol 271:E702-E710

4. Larsson O, Deeney JT, Branstrom R, Berggren P-O, Corkey BE (1996) Activation of the ATP-sensitive K + channel by long chain acyl-CoA. A role for modulation of pancreatic $\beta$-cell glucose sensitivity. J Biol Chem 271: 1062310626

5. Prentki M (1996) New insights into pancreatic $\beta$-cell metabolic signaling in insulin secretion. Eur J Endocrinology 134: 272-286

6. Brun T, Assimacopoulos-Jeannet F, Corkey BE, Prentki M (1997) Long-chain fatty acids inhibit acetyl-CoA carboxylase gene expression in the pancreatic $\beta$-cell line INS-1. Diabetes 46: 393-400

7. Assimacopoulos-Jeannet F, Thumelin S, Roche E et al. (1997) Fatty acids rapidly induce the carnitine palmitoyltransferase I gene in the pancreatic $\beta$-cell line INS-1. J Biol Chem 272: 1659-1664

8. Stein DT, Esser V, Stevenson B et al. (1996) Essentiality of circulating fatty acids for glucose- stimulated insulin secretion in the fasted rat. J Clin Invest 97: 2728-2735

9. Stein DT, Stevenson BE, Chester MW et al. (1997) The insulinotropic potency of fatty acids is profoundly influenced by their chain length and degree of saturation. J Clin Invest 100: 398-403

10. Sako Y, Grill V (1990) A $48 \mathrm{~h}$ lipid infusion in the rat timedependently inhibits glucose-induced insulin secretion and $\beta$-cell oxidation through a process likely coupled to fatty acid oxidation. Endocrinology 127: 1580-1589

11. Zhou Y-P, Grill V (1994) Long term exposure of rat pancreatic islets to fatty acids inhibits glucose-induced insulin secretion and biosynthesis through a glucose fatty acid cycle. J Clin Invest 93: 870-876

12. Zhou Y-P, Grill V (1996) A fatty acid induced decrease in pyruvate dehydrogenase activity is an important determinant of $\beta$-cell dysfunction in the obese $\mathrm{db} / \mathrm{db}$ mouse. Diabetes 45: 580-586 